\title{
NOTIONS OF STRONG ERGODICITY FOR STOCHASTIC ANALYSIS OF MULTIRATE SYSTEMS
}

\author{
Damián Marelli and Minyue Fu \\ School of Electrical Engineering and Computer Science \\ University of Newcastle, N.S.W. 2308 Australia
}

\begin{abstract}
For stochastic analysis of single-rate linear systems, a desirable property for stochastic signals is ergodicity in the mean and correlation. Unfortunately, as we show, the ergodicity property may not be preserved under downsampling and uniformly stable linear filtering. This poses a serious problem for stochastic analysis of multirate linear systems. In this paper, we introduce the notion of strong ergodicity which is preserved under a number of important multirate operations including downsampling, upsampling and time-variant uniformly stable linear filtering. We provide conditions for stochastic processes to be strongly ergodic. Using this result, we show that both independent random processes and bounded deterministic signals are strongly ergodic in the mean and correlation.
\end{abstract}

keywords: Ergodicity, Multirate systems, Stochastic analysis, Random processes.

\section{INTRODUCTION}

Multirate signal processing techniques find a wide range of applications; see, e.g., $[1,2,3]$. Two of the fundamental operations in a multirate system are downsampling and upsampling. Using these operations, signals can be decomposed into subbands where various signal processing operations take place. An example of such applications is the so-called subband adaptive filtering where filtering is done at individual subbands to gain a number of numerical advantages. Another example is the so-called subband system identification. In this technique, the input and output signals of a system to be identified are split into subbands by using filterbanks. Then, a parametric model is tuned in every subband. By doing so, better convergence and faster computation can be achieved in many cases. See, e.g., [4], [5], [6], [7].

In order to understand the statistical behavior of multirate systems, stochastic analysis is essential. For single-rate systems, the notion of ergodicity play a fundamental role in stochastic analysis. More specifically, random signals in the system are required to be ergodic in mean and/or correlation. When dealing with multirate systems, it is desirable that various stochastic analysis results can be carried over to subbands. This requires that random signals in each subband are required to be ergodic, which raises the following question: What conditions are required on the full-band signals so that the subband signals are ergodic? Unfortunately, as we will show, the ergodicity of a random signal may not be preserved under a number of operations, including downsampling and uniformly stable linear filtering.

Motivated by the discussion above, we look for suitable notions of ergodicity for multirate systems. More specifically, we introduce the notions of strong ergodicity in the mean and strong ergodicity in the correlation, which are properties preserved under a number of commonly used linear multirate operations, namely, downsampling, upsampling, addition and filtering by uniformly stable linear filters. We establish conditions for random processes to be strongly ergodic. Using these conditions, we show that white random processes with uniformly bounded second moments are strongly ergodic in the mean and that mutually independent random processes with uniformly bounded fourth moments are jointly strongly ergodic in the correlation.

These results mean that commonly used random processes in multirate linear systems are strongly ergodic (in the mean and correlation). Hence, many known stochastic analysis results available for single-rate systems can be readily applied to multirate systems.

Due to the space limitation, the proofs of results are not included in the paper.

\section{PRELIMINARIES}

In this section, we introduce the necessary notation and definitions for this paper.

\subsection{Random Processes}

Let $(\Omega, \mathcal{A}, \mathbb{P})$ be a probability space defined on a set $\Omega$ with $\sigma$ algebra $\mathcal{A}$ and probability measure $\mathbb{P}$. A random variable is an $\mathcal{A}$ measurable map $x: \Omega \rightarrow \mathbb{C}$, where $\mathbb{C}$ denotes the set of complex numbers. We denote the set of all random variables so defined by

$$
\mathcal{L}(\Omega, \mathcal{A}, \mathbb{P})=\{x: \Omega \rightarrow \mathbb{C}: x \text { is } \mathcal{A} \text {-measurable }\}
$$

The $p$-th (absolute) moment (or $p$-th norm) of a random variable $x$ is defined by

$$
\|x\|_{p}=\mathcal{E}\left\{|x|^{p}\right\}^{1 / p}
$$

where $\mathcal{E}\{\cdot\}$ denotes the expected value. We denote

$$
\mathcal{L}_{p}(\Omega, \mathcal{A}, \mathbb{P})=\left\{x \in \mathcal{L}(\Omega, \mathcal{A}, \mathbb{P}):\|x\|_{p}<\infty\right\}
$$

If $x, y \in \mathcal{L}_{2}(\Omega, \mathcal{A}, \mathbb{P})$, then their inner product is defined as

$$
\langle x, y\rangle=\mathcal{E}\left\{x^{*} y\right\}
$$

A (discrete-time) random process is a map $\xi: \mathbb{Z} \rightarrow \mathcal{L}(\Omega, \mathcal{A}, \mathbb{P})$, where $\mathbb{Z}$ is the set of integer numbers. We denote

$$
\mathcal{P}(\Omega, \mathcal{A}, \mathbb{P})=\{\xi: \mathbb{Z} \rightarrow \mathcal{L}(\Omega, \mathcal{A}, \mathbb{P})\}
$$

and

$$
\mathcal{P}_{p}(\Omega, \mathcal{A}, \mathbb{P})=\left\{\xi \in \mathcal{P}(\Omega, \mathcal{A}, \mathbb{P}): \sup _{t \in \mathbb{Z}}\|\xi(t)\|_{p}<\infty\right\}
$$


i.e., $\mathcal{P}_{p}(\Omega, \mathcal{A}, \mathbb{P})$ is a collection of random processes in $\mathcal{P}(\Omega, \mathcal{A}, \mathbb{P})$ with uniformly bounded $p$ th moments.

Since a deterministic signal can also be interpreted as a random process, the set $\mathcal{P}_{p}(\Omega, \mathcal{A}, \mathbb{P})$ includes deterministic signals in $l_{\infty}(\mathbb{Z})$

A random process is called white if its samples form a set of pairwise uncorrelated random variables, and it is called independent if its samples form a set of independent random variables. Also, a collection of random processes is called mutually independent if their samples form a set of independent random variables.

\subsection{Multirate Operations and Filtering}

Let $x \in \mathcal{P}(\Omega, \mathcal{A}, \mathbb{P})$ be a random process and $D \in \mathbb{N}$. The downsampled random process with downsampling rate $D$ is given by

$$
y(t)=x(D t)
$$

Similarly, the upsampled random process with upsampling rate $D$ is given by

$$
y(t)= \begin{cases}x(t / D) ; & t / D \in \mathbb{Z} \\ 0 ; & t / D \notin \mathbb{Z}\end{cases}
$$

A time-varying linear filter with impulse response $\left\{h_{t}(\tau)\right.$ : $t, \tau \in \mathbb{Z}\}$ is said to be uniformly stable if there exists $h \in l_{1}(\mathbb{Z})$ such that $\left|h_{t}(\tau)\right| \leq h(\tau), \forall t, \tau \in \mathbb{Z}$.

\subsection{Ergodicity}

A random process $x \in \mathcal{P}(\Omega, \mathcal{A}, \mathbb{P})$ is said to be ergodic in the mean if

$$
\lim _{T \rightarrow \infty} \frac{1}{T} \sum_{t=1}^{T} x(t)-\mathcal{E}\{x(t)\} \stackrel{w . \underline{p} .1}{=} 0
$$

Similarly, two random processes $x, y \in \mathcal{P}(\Omega, \mathcal{A}, \mathbb{P})$ are said to be jointly ergodic in the correlation if, for every $\tau \in \mathbb{Z}$,

$$
\lim _{T \rightarrow \infty} \frac{1}{T} \sum_{t=1}^{T} x^{*}(t) y(t+\tau)-\mathcal{E}\left\{x^{*}(t) y(t+\tau)\right\} \stackrel{w \cdot \underline{p} .1}{=} 0
$$

Also, a random process is ergodic in the correlation if it is jointly ergodic in the correlation with itself.

\section{MOTIVATING EXAMPLES}

In this section we show via examples that ergodicity in the correlation may not be preserved under a number of linear multirate operations. These operations include downsampling, linear filtering and addition.

Example 1 Consider the probability space $([-1 / 2,1 / 2], \mathcal{B}, \lambda)$, where $\mathcal{B}$ denotes the Borel $\sigma$-algebra on the set $[-1 / 2,1 / 2]$ and $\lambda$ denotes the Lebesgue measure. Define the random process $x \in$ $\mathcal{P}([-1 / 2,1 / 2], \mathcal{B}, \lambda)$ as follows:

$$
(x(t))(\omega)= \begin{cases}\frac{1}{2}(1+\operatorname{sign}(\omega)) ; & t \text { is even } \\ \frac{1}{2}(1-\operatorname{sign}(\omega)) ; & t \text { is odd }\end{cases}
$$

where $\operatorname{sign}(\omega)=|\omega| / \omega$. Then,

$$
\begin{aligned}
& \left.\frac{1}{T} \sum_{t=1}^{T} x^{*}(t)\right)(\omega)(x(t+\tau))(\omega)-\mathcal{E}\left\{\left(x^{*}(t)\right)(\omega)(x(t+\tau))(\omega)\right\} \\
& \quad= \begin{cases}\frac{1}{T} \sum_{t=1}^{T} \frac{(-1)^{t+1}}{2} \operatorname{sign}(\omega) ; & \tau \text { is even } \\
0 ; & \text { is odd }\end{cases} \\
& \quad \rightarrow \quad 0, \text { as } T \rightarrow \infty
\end{aligned}
$$

so $x$ is ergodic in the correlation. Let $y \in \mathcal{P}([-1 / 2,1 / 2], \mathcal{B}, \lambda)$ be generated from $x$ by downsampling by a factor of 2 , i.e.,

$$
(y(t))(\omega)=(x(2 t))(\omega)=\frac{1}{2}(1+\operatorname{sign}(\omega))
$$

Then,

$$
\begin{aligned}
& \left.\left.\frac{1}{T} \sum_{t=1}^{T} y^{*}(t)\right)(\omega)(y(t+\tau))(\omega)-\mathcal{E}\left\{\left(y^{*}(t)\right)(\omega)(y(t+\tau))(\omega)\right\}\right] \\
& \quad=\frac{1}{2} \operatorname{sign}(\omega) \nrightarrow 0, \quad \text { as } \quad T \rightarrow \infty
\end{aligned}
$$

This implies that $y$ is not ergodic in the correlation.

Example 2 Consider the random process $x$ in Example 1 and the uniformly stable time-variant linear filter with the following impulse response

$$
h_{t}(\tau)= \begin{cases}\delta(\tau) ; & t \text { is even } \\ \delta(\tau-1) ; & t \text { is odd }\end{cases}
$$

where $\delta(\tau)$ is the Kronecker function. Let $y$ be the random process obtained by filtering $x$ through $h_{t}(\tau)$, i.e.,

$$
(y(t))(\omega)=\sum_{\tau=-\infty}^{\infty} h_{t}(\tau)(x(t-\tau))(\omega)=\frac{1}{2}(1+\operatorname{sign}(\omega))
$$

Then,

$$
\begin{aligned}
& \left.\frac{1}{T} \sum_{t=1}^{T} y^{*}(t)\right)(\omega)(y(t+\tau))(\omega)-\mathcal{E}\left\{\left(y^{*}(t)\right)(\omega)(y(t+\tau))(\omega)\right\} \\
& \quad=\frac{1}{2} \operatorname{sign}(\omega) \nrightarrow 0, \text { as } T \rightarrow \infty
\end{aligned}
$$

which means that $y$ is not ergodic in the correlation.

Example 3 Consider the following random processes in $\mathcal{P}([-1 / 2$, $1 / 2], \mathcal{B}, \lambda)$ :

$$
\begin{aligned}
& (x(t))(\omega)=\operatorname{sign}(\omega) ; \\
& (y(t))(\omega)=1
\end{aligned}
$$

It is easy to verify that, for all $\tau \in \mathbb{Z}, T \in \mathbb{N}$,

$$
\begin{aligned}
& \frac{1}{T} \sum_{t=1}^{T}\left[x^{*}(t)\right)(\omega)(x(t+\tau))(\omega) \\
& \left.-\mathcal{E}\left\{\left(x^{*}(t)\right)(\omega)(x(t+\tau))(\omega)\right\}\right]=0 ; \\
& \frac{1}{T} \sum_{t=1}^{T}\left[y^{*}(t)\right)(\omega)(y(t+\tau))(\omega) \\
& -\mathcal{E}\left\{\left(y^{*}(t)\right)(\omega)(y(t+\tau))(\omega)\right\}=0
\end{aligned}
$$

Hence, $x$ and $y$ are ergodic in the correlation. Define

$$
(z(t))(\omega)=(x(t))(\omega)+(x(t))(\omega)=1+\operatorname{sign}(\omega)
$$

It is easy to verify that

$$
\begin{aligned}
& \frac{1}{T} \sum_{t=1}^{T}\left[z^{*}(t)\right)(\omega)(z(t+\tau))(\omega) \\
& \left.-\mathcal{E}\left\{\left(z^{*}(t)\right)(\omega)(z(t+\tau))(\omega)\right\}\right] \\
= & \operatorname{sign}(\omega) \not \rightarrow 0, \text { as } T \rightarrow \infty
\end{aligned}
$$

Hence, $z$ is not ergodic in the correlation. 


\section{STRONG ERGODICITY}

In this section, we introduce the notions of strong ergodicity in the mean and in the correlation.

\subsection{Strong Ergodicity in the Mean}

Definition 1 The random process $x \in \mathcal{P}(\Omega, \mathcal{A}, \mathbb{P})$ is called strongly ergodic in the mean if the following conditions hold

(M1) $x$ is ergodic in the mean.

(M2) If the random process $y \in \mathcal{P}(\Omega, \mathcal{A}, \mathbb{P})$ is also strongly ergodic in the mean, then $x+y$ is strongly ergodic in the mean.

(M3) The filtering of $x$ by a uniformly stable linear filter, yields a random process that is strongly ergodic in the mean.

(M4) The downsampling of $x$ by any factor yields a random process that is strongly ergodic in the mean.

(M5) The upsampling of $x$ by any factor yields a random process that is strongly ergodic in the mean.

\subsection{Strong Ergodicity in the Correlation}

Our aim here is to give a definition for strong ergodicity in the correlation in a way similar to that of strong ergodicity in the mean. However, this can not be done straightforwardly. Recall that Condition (M2) says that the addition of two signals which are strongly ergodic in the mean yields a signal which is also strongly ergodic in the mean. However, in the example below, we show that the addition of two independent signals can yield a non-ergodic signal. Imposing a condition similar to (M2) would exclude a large class of important random processes. Therefore, we need to relax the requirements for strong ergodicity in the correlation.

Example 4 Consider the probability space $([0,1), \mathcal{B}, \lambda)$. For every $\omega \in[0,1)$, let its binary expansion be

$$
\omega=\sum_{t=1}^{\infty} \omega_{t} 2^{-t}
$$

Define the random process $x \in \mathcal{P}([0,1), \mathcal{B}, \lambda)$ by

$$
(x(t))(\omega)=2 \omega_{t}-1
$$

Define $y \in \mathcal{P}([0,1), \mathcal{B}, \lambda)$ by

$$
(y(t))(\omega)=\left\{\begin{array}{cc}
(x(t))(\omega) ; \quad \omega \in\left[0, \frac{1}{2}\right) \\
-(x(t))(\omega) ; \quad \omega \in\left[\frac{1}{2}, 1\right)
\end{array}\right.
$$

It is easy to verify that $x$ and $y$ are independent, see [8, Example 4, p.56], however they are not mutually independent. Let $z \in$ $\mathcal{P}([0,1), \mathcal{B}, \lambda)$ be defined by

$$
\begin{aligned}
(z(t))(\omega) & =(x(t))(\omega)+(y(t))(\omega) \\
& = \begin{cases}2(x(t))(\omega) ; & \omega \in\left[0, \frac{1}{2}\right) \\
0 ; & \omega \in\left[\frac{1}{2}, 1\right)\end{cases}
\end{aligned}
$$

Then,

$$
\begin{aligned}
& \frac{1}{T} \sum_{t=1}^{T}\left(z^{*}(t)\right)(\omega)(z(t))(\omega)-\mathcal{E}\left\{\left(z^{*}(t)\right)(\omega)(z(t))(\omega)\right\} \\
= & \begin{cases}2 ; & \omega \in\left[0, \frac{1}{2}\right) \\
0 ; & \omega \in\left[\frac{1}{2}, 1\right)\end{cases}
\end{aligned}
$$

Therefore, $z$ is not ergodic in the correlation.
To get around the difficulty mentioned above, we observe that there is a property of addition for joint ergodicity, i.e., the addition of two signals which are ergodic in the correlation and jointly ergodic in the correlation yields an ergodic signal. In view of this, we introduce the notion of joint strong ergodicity which has a similar property.

Definition 2 Two random processes $x, y \in \mathcal{P}(\Omega, \mathcal{A}, \mathbb{P})$ are said to be jointly strongly ergodic in the correlation if the following conditions hold:

(CO) $y$ and $x$ are jointly strongly ergodic, i.e., the condition is symmetric.

(C1) $x$ and $y$ are jointly ergodic in the correlation.

(C2) If the random process $z \in \mathcal{P}(\Omega, \mathcal{A}, \mathbb{P})$ is jointly strongly ergodic in the correlation with both $x$ and $y$, then $z$ is also jointly strongly ergodic in the correlation with $x+y$.

(C3) The filtering of y by a uniformly stable linear system, yields a random process that is jointly strongly ergodic in the correlation with $x$.

(C4) The downsampling of $y$ by any factor yields a random process that is jointly strongly ergodic in the correlation with $x$.

(C5) The upsampling of y by any factor yields a random process that is jointly strongly ergodic in the correlation with $x$.

A random process is called strongly ergodic in the correlation if it is jointly strongly ergodic in the correlation with itself.

\section{MAIN RESULTS}

In this section, we provide our main results. We first introduce a norm on a subset of random processes in $\mathcal{P}_{2}(\Omega, \mathcal{A}, \mathbb{P})$, which characterizes the decay rate of the autocorrelation function of the random process. We then use this subset to give sufficient conditions for strong ergodicity in the mean and in the correlation. In particular, a white random process with uniformly bounded second moments is strongly ergodic in the mean and two mutually independent random processes with uniformly bounded fourth moments are jointly strongly ergodic in the correlation.

Definition 3 Let $\xi \in \mathcal{P}_{2}(\Omega, \mathcal{A}, \mathbb{P})$ be a random process and $T \in$ $\mathbb{N}$. Define

$$
\|\xi\|_{\mathcal{S}}=\sup _{T \in \mathbb{N}, d \in \mathbb{Z}}\left(\frac{1}{T} \sum_{t, s=1}^{T}|\langle\xi(s+d), \xi(t+d)\rangle|^{2}\right)^{1 / 4}
$$

and

$$
\mathcal{S}(\Omega, \mathcal{A}, \mathbb{P})=\left\{\xi \in \mathcal{P}_{2}(\Omega, \mathcal{A}, \mathbb{P}):\|\xi\|_{\mathcal{S}}<\infty\right\}
$$

Lemma 1 The map $\|\cdot\|_{\mathcal{S}}: \mathcal{S}(\Omega, \mathcal{A}, \mathbb{P}) \rightarrow \mathbb{R}^{+}$defines a norm on $\mathcal{S}(\Omega, \mathcal{A}, \mathbb{P})$, i.e., for any $\xi, \zeta \in \mathcal{S}(\Omega, \mathcal{A}, \mathbb{P})$ and $c \in \mathbb{C}$, the following conditions hold:

(N1) $\|c \xi\|_{\mathcal{S}}=|c|\|\xi\|_{\mathcal{S}} ;$

(N2) $\|\xi+\zeta\|_{\mathcal{S}} \leq\|\xi\|_{\mathcal{S}}+\|\zeta\|_{\mathcal{S}}$;

(N3) $\|\xi\|_{\mathcal{S}}=0 \Rightarrow \xi=0$. 
Lemma 2 Let $\xi \in \mathcal{P}(\Omega, \mathcal{A}, \mathbb{P})$ be random process and let $\zeta$ be a random process generated from $\xi$ by downsampling, upsampling or filtering via a uniformly stable linear filter. If $\xi \in \mathcal{S}(\Omega, \mathcal{A}, \mathbb{P})$, then $\zeta \in \mathcal{S}(\Omega, \mathcal{A}, \mathbb{P})$.

Lemma 3 Let $x \in \mathcal{P}_{1}(\Omega, \mathcal{A}, \mathbb{P})$ be a random process. If the random processes $\xi_{x}$ defined by

$$
\xi_{x}(t)=x(t)-\mathcal{E}\{x(t)\}
$$

is in $\mathcal{S}(\Omega, \mathcal{A}, \mathbb{P})$, then $x$ is strongly ergodic in the mean.

Theorem 1 If the random process $x \in \mathcal{P}_{2}(\Omega, \mathcal{A}, \mathbb{P})$ is white, then it is strongly ergodic in the mean.

Putting together the ideas of this section, we have the following result, which we state without proof.

Theorem 2 Let $v$ be a random process formed from the random processes of the finite collection $u_{m} \in \mathcal{P}_{2}(\Omega, \mathcal{A}, \mathbb{P}), m=1, \cdots$, $M$, by any finite combination of additions, filtering with uniformly stable linear filters, downsampling and upsampling. If for every $m$, the random process $u_{m}$ is white, then $v$ is ergodic in the mean.

Remark 1 Note that the signals $u_{m}$ in Theorem 4 can include deterministic signals in $l_{\infty}(\mathbb{Z})$ because they are also in $\mathcal{P}_{2}(\Omega, \mathcal{A}, \mathbb{P})$.

Lemma 4 Let $x, y \in \mathcal{P}_{1}(\Omega, \mathcal{A}, \mathbb{P})$ be random processes. If the collection of random processes $\xi_{x, y}(A, B, a, b),(A, B, a, b) \in$ $\mathbb{N} \times \mathbb{N} \times \mathbb{Z} \times \mathbb{Z}$, defined by

$$
\begin{aligned}
& \xi_{x, y}(A, B, a, b)(t) \\
= & x^{*}(A t+a) y(B t+b)-\mathcal{E}\left\{x^{*}(A t+a) y(B t+b)\right\}
\end{aligned}
$$

are in $\mathcal{S}(\Omega, \mathcal{A}, \mathbb{P})$ and the norms $\left\|\xi_{x, y}(A, B, a, b)\right\|_{\mathcal{S}}$ are uniformly bounded, then $x$ and $y$ are jointly strongly ergodic in the correlation.

Theorem 3 Let $x, y \in \mathcal{P}_{4}(\Omega, \mathcal{A}, \mathbb{P})$ be random processes. Then:

(i) If $x$ is independent, then it is strongly ergodic in the correlation.

(ii) If $x$ and $y$ are mutually independent, then $x$ and $y$ are jointly strongly ergodic in the correlation.

Theorem 4 Let $v$ be a random process formed from the random processes of the finite collection $u_{m} \in \mathcal{P}_{4}(\Omega, \mathcal{A}, \mathbb{P}), m=1, \cdots$, $M$, by any finite combination of additions, filtering with uniformly stable linear filters, downsampling and upsampling. If the random processes $\left\{u_{m}: m=1, \cdots, M\right\}$ are mutually independent, then $v$ is ergodic in the correlation.

Remark 2 Note that the signals $u_{m}$ in Theorem 4 can include deterministic signals in $l_{\infty}(\mathbb{Z})$ because they are also in $\mathcal{P}_{4}(\Omega, \mathcal{A}, \mathbb{P})$. Also note that if a family of random processes have a Gaussian distribution, then the uniform boundedness of the fourth moments is equivalent to that of the second moments.

In Example 1, we showed an random process, which is ergodic in the correlation, whose ergodicity was lost after downsampling and linear filtering. By definition, this random process is not strongly ergodic in the correlation. We show below that this random process indeed violates the condition in Lemma 4.
Example 5 Consider the random process $x$ in Example 1. We have

$$
\left|\mathcal{E}\left\{\xi_{x, x}^{*}(1,1,0,0)(t) \xi_{x, x}(1,1,0,0)(s)\right\}\right|=1, \quad \forall t, s \in \mathbb{N}
$$

It follows that

$$
\frac{1}{T} \sum_{t, s=1}^{T}\left|\mathcal{E}\left\{\xi_{x, x}^{*}(1,1,0,0)(t) \xi_{x, x}(1,1,0,0)(s)\right\}\right|^{2}=T
$$

and

$$
\left\|\xi_{x, x}(1,1,0,0)\right\|_{\mathcal{S}}=\infty
$$

Therefore, the condition in Lemma 4 is violated.

\section{CONCLUSION}

In this paper we have introduced the notions of strong ergodicity in the mean and in the correlation which are suitable for stochastic analysis of multirate systems. The definitions of these notions are motivated by the fact that the ergodicity (in the mean or correlation) may not be preserved under several linear and multirate operations. In contrast, strong ergodicity in the mean or correlation implies ergodicity in the mean or correlation, respectively, and they are invariant under the transformations involved in a multirate linear system, i.e., time-variant, uniformly stable linear filtering, downsampling, upsampling and addition (In the case of strong ergodicity in the correlation, the signals to be added need also be jointly strongly ergodic in the correlation). We have also shown that independent random processes, which includes bounded deterministic signals, are strongly ergodic in the mean or correlation and mutually independent random processes are jointly strongly ergodic in the correlation. Therefore, all the signals generated from these by the transformations mentioned above are strongly ergodic in the mean or correlation. As a consequence, the usual signals of interest are strongly ergodic in the mean or correlation.

\section{REFERENCES}

[1] P. P. Vaidyanathan, Multirate Systems and Filterbanks, Prentice Hall, Englewood Cliffs, N. J., 1993.

[2] R. E. Crochiere and L. R. Rabiner, Multirate digital signal processing, Prentice Hall, Englewood Cliffs, N. J., 1983.

[3] M. Vetterli and J. Kovačević, Wavelets and Subband Coding, Prentice Hall, Englewood Cliffs, N. J., 1995.

[4] A. Gilloire and M. Vetterlli, "Adaptive filtering in subbands with critical sampling: analysis, experiments, and application to acoustic echo cancellation," IEEE Transactions on Signal Processing, vol. 40, no. 8, pp. 1862-1875, August 1992.

[5] Y. Lu and J. Morris, "Gabor expansion for adaptive echo cancellation," IEEE Signal Proc. Magazine, vol. 16, no. 2, pp. 6880, March 1999.

[6] D. Marelli and M. Fu, "Asymptotic properties of subband identification," to appear in IEEE Trans. Signal Proc..

[7] D. Marelli and M. Fu, "Performance analysis for subband identification," to appear in IEEE Trans. Signal Proc.

[8] K. L. Chung, A course in probability theory, Academic Press, New York, 2nd edition, 1974. 